\title{
Introduction to the special issue of best papers from RE2015 conference
}

\author{
Didar Zowghi $^{1} \cdot$ Vincenzo Gervasi $^{2}$
}

Published online: 6 May 2016

(c) Springer-Verlag London 2016

The 23rd IEEE International Requirements Engineering conference (RE'15) was held on August 24-28, 2015, in the beautiful city of Ottawa, Canada, hosted by the General Chair Daniel Amyot from the University of Ottawa. This conference is the premier international requirements engineering forum for researchers and practitioners to present and discuss their most recent innovations and experiences. To continue the long-standing collaboration between the RE conference and the Requirements Engineering Journal, this special issue presents extended versions of four selected papers from RE' 15 . We invited the authors of the best papers presented at the conference to submit extended versions of their papers to reach out the wider journal readership. As the program co-chairs of RE'15, it is our pleasure to provide an introduction to these papers.

The RE'15 theme was Requirements for the masses, requirements from the masses encouraging submissions of innovative research addressing the specific problems of massive-scale RE. Three out of 4 papers published in this special issue are related to massive RE. It is encouraging to report that the main theme of the conference was taken seriously! The conference offered a rich technical program, including a number of selected papers from (and for) both academic and industrial perspectives. In addition, this year we introduced a new track named "RE: Next!" to present shorter previews of ongoing work. This track provided an excellent opportunity to hear what people around the world

\section{Didar Zowghi}

Didar.Zowghi@uts.edu.au

1 Faculty of Engineering and Information Technology, University of Technology, Sydney, Australia

2 Dipartimento di Informatica, University of Pisa, 56127 Pisa, Italy are currently working on, to establish early collaborations, to stimulate ideas and to share experiences.

RE'15 conference hosted three excellent keynote speakers. Aydin Mirzaee (General Manager, FluidReview, Survey Monkey) told the story of how FluidReview and then SurveyMonkey managed their requirements and product roadmap, in a context where the company has tens of millions of users and stakeholders. Karen Holtzblatt (Founder \& CEO of InContext) discussed Contextual Design, a set of design principles based on a deep understanding of users' contexts. Last but not least Michael Jackson (Independent Consultant and Visiting Research Professor at the Open University, UK) shared his insightful reflections about how $\mathrm{RE}$ can be rethought in terms of meeting the needs and desires of large numbers of stakeholders in cyber physical systems.

We received submissions of 500 authors, from 28 different countries. Overall, 16 different countries were represented in the main conference program, with many other countries represented in the various workshops and other events. RE'15 attracted 171 full papers in total (97 Research track, 23 Industry track, 51 RE:Next), and the four papers published here are extended versions of the best from the 97 research submissions. Papers in the research track were independently blind reviewed by three members of the Program Committee, followed by a period of online discussion open to all PC members. All papers that had at least one positive review at the end of the discussion period were examined at the Program Committee Meeting that was held in Pisa, Italy, on May 14 and 15. From the research track category, 19 papers were finally accepted for presentation at the conference (an acceptance rate of $19.6 \%$ ). We extend our appreciation to the great effort exerted by the members of the Program Committee in dedicating their time to provide valuable reviews. We 
also express our appreciation to the members of the Organizing Committee of RE'15: without their help and expertise, the conference would not have been successful.

Each of the four extended papers in this issue went through additional rigorous review by three reviewers according to the REJ review process before finally being accepted for publication.

The first paper in this special issue entitled "On the automatic classification of app reviews" is authored by Walid Maalej, Zijad Kurtanović, Hadeer Nabil and Christoph Stanik. The paper was one of the two to receive a best paper award at the conference, and has addressed the automation of the classification of user reviews for mobile apps by extracting the useful information from the large amount of available comments and ratings. The authors have employed several techniques (review metadata, text classification, natural language processing and sentiment analysis) for classifying the reviews in four types: bug report, feature requests, user experience and text ratings. They carried out a series of experiments to evaluate the accuracy of the techniques and compared them with simple string matching. Their results indicate that the metadata alone provide low accuracy, whereas when combined with simple text classification and natural language processing, the classification precision was raised up to $88-90 \%$ and the recall up to $90-99 \%$. The results assisted them in designing a review analytical tool for vendors and developers of apps to extract useful information from the large amount of scattered reviews. The paper also presents the main features of the tool and summary of the 9 interviews with practitioners on the usefulness of the analytics tool in practice.

The second best paper award was presented at the conference to the paper entitled "Ambiguity and tacit knowledge in requirements elicitation interviews" by Alessio Ferrari, Paola Spoletini and Stefania Gnesi. The paper addresses the long recognized issue of ambiguity in requirements engineering. They focus specifically on ambiguity in requirements elicitation interviews when requirements are in tacit form of ideas and yet to be discovered in clear form. In order to study ambiguity in this scenario, they have conducted 34 customer-analyst interviews. The authors defined a framework to categorize ambiguity into four sub-phenomena, i.e., unclarity, multiple understanding, incorrect disambiguation and correct disambiguation. Their study has revealed the peculiar relation between ambiguity and tacit knowledge. They have presented supporting examples from the interviews to show how ambiguity can be considered as a resource for discovering the tacit knowledge.

The third selected paper for this special issue entitled "Detecting, classifying and tracing non-functional software requirements" by Anas Mahmoud and Grant Williams describes an unsupervised approach for detecting, classifying and tracing non-functional requirements (NFRs). Their proposed approach employs textual semantics of software functional requirements (FRs) to infer the quality constraints in the system. They have conducted a systematic analysis of a series of word similarity methods and clustering techniques to generate semantically cohesive clusters of FR words and then classified them into different categories of NFRs by comparing their semantic similarity to basic NFR labels. A traceability of discovered NFRs was conducted in the source code artefacts. From the experiments on three systems, the results show that methods that exploit massive sources of textual human knowledge are more accurate in capturing and modeling the notion of similarity between FR words in a software system.

The fourth paper entitled "Improving agile requirements: The quality user story framework and tool" is authored by Garm Lucassen, Fabiano Dalpiaz, Jan Martijn E. M. Van der Werf and Sjaak Brinkkemper. They have addressed the problem of poor quality of user stories in agile development. They have proposed a Quality User Story (QUS) Framework which is a set of 13 quality criteria for user story writers. These criteria have been incorporated in Automatic Quality User Story Artisan (AQUSA) software tool. The tool uses natural language processing techniques to detect quality defects and suggest possible remedies. The paper presents the implementation of AQUSA and provides an evaluation report by analyzing 1023 user stories from 18 software companies. The results are deemed promising, and the authors have discussed future improvements.

We extend a special thank to the authors of the invited papers as well as the reviewers of these papers. Finally, we are grateful to Peri Loucopoulos, the Editor in Chief of REJ, and the REJ editorial team for their assistance in the preparation of this issue and for continued support and collaboration between RE conference and REJ.

RE'16 edition will take place in Beijing, China, and RE'17 will return to Europe to be held in Lisbon, Portugal. We cordially invite all the readers of REJ to join us for both conferences. ${ }^{1}$

\footnotetext{
${ }^{1}$ For more information on past and upcoming RE conferences, please visit: http://requirements-engineering.org/.
} 